
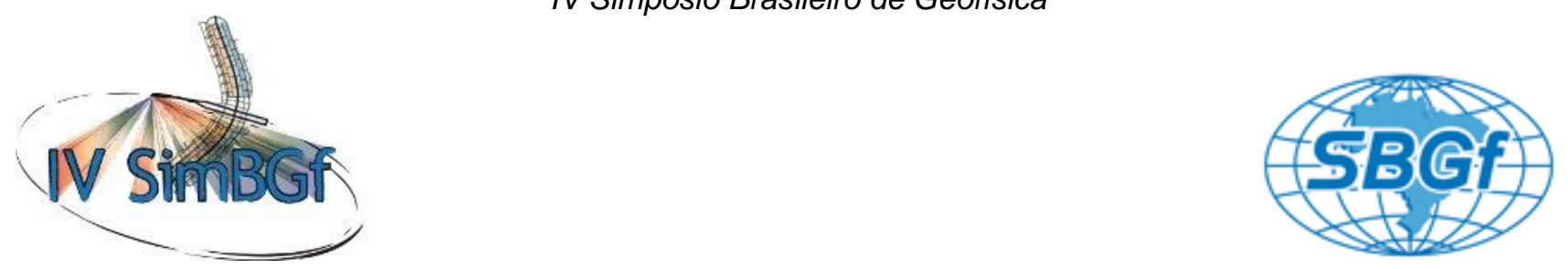

\title{
Estimativa de parâmetros elásticos a partir das amplitudes sísmicas de ondas refletidas P-P P-Sv Sv-P e Sv-Sv: Parte II. Analise do problema de inversão não linear.
}

Oleg Bokhonok", Liliana Alcazar Diogo**, Renato Luiz Prado**, *IAG/USP - Programa de Pós-graduação, ${ }^{*} \mid A G / U S P$ Departamento de Geofísica

Copyright 2010, SBGf - Sociedade Brasileira de Geofísica

Este texto foi preparado para a apresentação no IV Simpósio Brasileiro de Geofísica Brasília, 14 a 17 de novembro de 2010. Seu conteúdo foi revisado pelo Comitê Técnico do IV SimBGf, mas não necessariamente representa a opinião da SBGf ou de seus associados. É proibida a reprodução total ou parcial deste material para propósitos comerciais sem prévia autorização da SBGf.

\section{Resumo}

This paper shows a numerical study aiming to predict the "real values" of seismic velocities (Vp, Vs) and densities from non linear AVO analysis of multicomponent shallow seismic data. The amplitudes were modeled by Zoeppritz equations. To optimize the least squares of observed and computed amplitudes we used parallel Multi-Start run for Controlled Random Search algorithm (CRS) and kernel density estimation. We present a new approach for multidimensional objective function analysis, with numerous implications for the accuracy and efficiency of non-linear inversion.

\section{Introdução}

O problema inverso investigado, descrito em Bokhonok et al (2010), trata-se de um problema não linear e mal posto. Resumidamente, propõem-se a determinação de 6 parâmetros (densidades $(\rho)$ e velocidades das ondas $P$ ( $\alpha$ ) e $S(\beta)$, acima e abaixo da interface refletora) a partir da inversão das amplitudes das reflexões sísmicas multicomponente, modeladas pelas equações de Zoeppritz para ondas refletidas PP, PSv, SvP e SvSv.

Uma análise das características da função objetivo é indispensável para a escolha do método de otimização adequado para a solução do problema inverso. É importante lembrar que no caso dos problemas de inversão mal postos, várias soluções (modelos) podem representar igualmente bem os dados (amplitudes das reflexões sísmicas). Portanto, a convergência de um algoritmo de otimização global para o ponto de mínimo pode não garantir a solução correta do problema inverso.

Neste trabalho é detalhado como o próprio método de otimização foi empregado para avaliar as características multidimensionais das funções objetivo, tais como: linearidade, mínimos locais, ambigüidades, etc. Foram sugeridos critérios que podem ser adotados para avaliar a performance do algoritmo de busca e garantir confiabilidade à estimativa dos parâmetros alcançada. Alguns dos resultados deste analise estão apresentados a seguir, para exemplificar a metodologia proposta.

\section{Procedimento de otimização}

A busca do mínimo global da função objetivo é realizada com a implementação de um procedimento do tipo MultiStart, usando um algoritmo estocástico de otimização global CRS (Controlled Random Search) (Price, 1979, Price, 1983, Eligius et al, 2001).

Um espaço inicial de busca é definido para se gerar um conjunto aleatório de pontos iniciais (pontos start) uniformemente distribuídos. Entretanto, durante a execução do procedimento de otimização, o espaço de busca é ilimitado.

Algoritmos estocásticos quando executados mais de uma vez raramente convergem para o mesmo ponto. A realização do procedimento de busca diversas vezes produz um conjunto de soluções que formam uma distribuição. No caso da inversão dos coeficientes de reflexão usando mínimos quadrados obtém-se uma distribuição normal: o valor da média aritmética é igual à máxima verossimilhança desta distribuição. Por isso neste caso é aceitável utilizar a média ou a mediana para a estimativa final dos parâmetros. Normalmente, a mediana é considerada mais robusta (Isaaks, 1989).

\section{Análise das soluções encontradas}

Os testes foram realizados usando os parâmetros do modelo numérico de Pullan \& Hunter (1985) (Tabela 1).

Tabela 1 - Parâmetros de modelo numérico
\begin{tabular}{|c|c|}
\hline$\alpha_{1}$ & $1500 \mathrm{~m} / \mathrm{s}$ \\
\hline$\alpha_{2}$ & $3750 \mathrm{~m} / \mathrm{s}$ \\
\hline$\beta_{1}$ & $452 \mathrm{~m} / \mathrm{s}$ \\
\hline$\beta_{2}$ & $2165 \mathrm{~m} / \mathrm{s}$ \\
\hline$\rho_{1}$ & $1530 \mathrm{~kg} / \mathrm{m}^{3}$ \\
\hline$\rho_{2}$ & $2430 \mathrm{~kg} / \mathrm{m}^{3}$ \\
\hline$h$ & $50 \mathrm{~m}$ \\
\hline
\end{tabular}

A representação gráfica do conjunto de soluções das diversas realizações do processo de otimização é definida aqui por "mapas de dispersão". 
O critério inicial para avaliar os conjuntos de dispersão é o caráter da distribuição normal bivariada apresentado nos mapas, ou seja, quanto melhor está otimizado um par de parâmetros maior é a correlação entre suas distribuições. Já no caso de um fiasco, a distribuição é representada por duas distribuições normais independentes. Como exemplo, na Figura 1-a é apresentado o mapa de dispersão dos resultados da otimização para a obtenção dos parâmetros de densidade usando-se o coeficiente de reflexão da onda SvSv para ângulos de incidência de 0 a 30 graus; e na Figura 1-b, os resultados obtidos para ângulos de incidência de 30 a 60 graus. As soluções obtidas pela otimização estão separadas em faixas de valores dos mínimos encontrados, marcadas por cores diferentes. A faixa que corresponde aos menores valores do mínimo (vermelho) é escolhida para determinar o valor do parâmetro estimado. Nota-se que os resultados apresentados na Figura 1-a indicam que a faixa vermelha é a melhor correlacionada. Já na Figura 1-b, a distribuição bivariada é representada por duas distribuições normais independentes, indicando que não há confiabilidade no resultado final para a estimativa dos parâmetros. Esses resultados concordam com a análise de sensibilidade apresentada na primeira parte deste trabalho (Bokhonok et al, 2010).

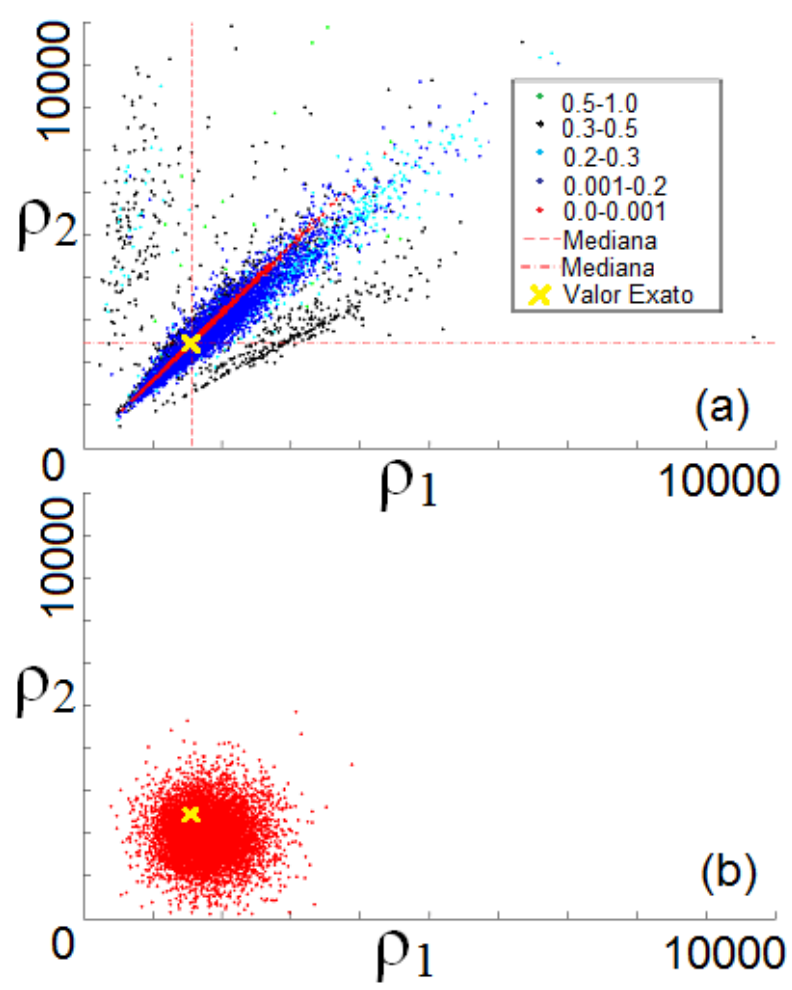

Figura 1 - Mapas de dispersão para as densidades otimizadas usando coeficiente de reflexão de onda SvSv: (a) de 0 a 30 graus; (b) de 30 a 60 graus.
$\mathrm{Na}$ Figura 2 são apresentados varios exemplos que foram selecionados para ilustrar algumas das utilidades da análise dos mapas de dispersão para avaliar diferentes aspectos da estratégia de otimização, tais como:

- A performance de diferentes procedimentos de busca: são apresentados os resultados obtidos com a função fminsearch do aplicativo MatLab (Figura 2-a), e com a implementação adaptada para o procedimento CRS (Figura 2-c). Os dois métodos são baseados no conceito de SIMPLEX, mas com algumas notáveis diferenças de implementação. Por exemplo, no caso de fminsearch a reflexão em relação ao centróide é do ponto que encontra o maior (pior) valor de função objetivo, já o CRS faz a escolha deste ponto ao acaso (randomicamente). Sendo assim fminsearch converge mais rapidamente, e um número maior de vezes, ao mínimo local, como pode ser claramente observado nas Figuras 2-a (fminsearch) e 2-c (CRS).

- A influência do tamanho do espaço inicial de busca: comparando as distribuições das soluções obtidas pelas múltiplas otimizações da função objetivo do coeficiente de reflexão PSv com o algoritmo CRS, utilizando um espaço inicial maior (Figura 2-b) e menor (Figura 2-d), nota-se que um maior espaço de busca inicial gera maior dispersão das soluções em torno da ambigüidade global. Conseqüentemente, um número maior de otimizações é necessário para se conseguir uma distribuição adequada para a análise estatística da solução final.

- A comparação entre diferentes funções objetivo: como exemplificado para a estimativa das velocidades da onda $\mathrm{P}$, utilizando-se os coeficientes de reflexão PP (Figura 2-e), PSv (Figura 2-g) e PP-PSv em conjunto (Figura 2-i), nota-se que a função objetivo conjunta apresenta a menor complexidade;

- O efeito do ruído nos dados: essa análise foi feita adicionando-se ruído randômico gaussiano nas curvas dos coeficientes de reflexão PP e PSv. Comparando-se os resultados para os dados sem e com ruído, observase uma maior dispersão das soluções em torno da faixa de ambigüidade global para os dados com ruído, como exemplificado para os coeficientes de reflexão: PP (Figuras 2-e sem ruído e 2-f com ruído), PSv (Figuras 2-g sem ruído e 2-h com ruído) e PP-PSv (Figuras 2-i sem ruído e 2-j com ruído). Neste contexto cresce a importância do uso da função objetivo conjunta.

Os mapas de dispersão também podem ser analisados pelo método de agrupamento por núcleo de densidade (kernel density estimation) implementado por Botev, et al. (2010). Como exemplo, nota-se na Figura 3 que a comparação entre as funções objetivo: PP, PSv e PPPSv para dados com ruído é mais evidente quando analisada pelo método de agrupamento por núcleo de densidade do que no último exemplo citado acima (Figuras 2-f, 2-h e 2-j). É observado um aumento de densidade em torno de ambigüidade global para função objetivo conjunta (PP-PSv). 
A análise do tamanho do espaço de busca pelo método de agrupamento por núcleo de densidade aplicado nos mapas de dispersão pode ser muito útil para a solução do problema inverso proposto. Sabe-se que a otimização de uma função partindo de um ponto perto do mínimo global tende a convergir melhor. Quando não existe uma boa informação a priori sobre o meio investigado, os pontos para iniciar a busca são gerados randomicamente e de modo uniforme dentro do espaço de busca. Quando o espaço é muito grande, são poucos os pontos que conseguem convergir para as proximidades do mínimo global e, assim, é mais difícil conseguir uma boa distribuição para proceder a análise estatística para a estimativa dos parâmetros do modelo.

Após a obtenção de um conjunto inicial de soluções de mínimo, da análise da dispersão destes pontos, pode-se reduzir o espaço para gerar os pontos iniciais de busca. Para tal é usado o método de agrupamento por núcleo de densidade. Este procedimento é repetido tantas vezes quanto necessário até se obter um espaço onde o algoritmo de busca converge com facilidade. O objetivo é conseguir uma boa distribuição de pontos em torno do mínimo global que permita a determinação dos valores dos parâmetros usando a mediana. A Figura 4 ilustra o uso do método de agrupamento por núcleo de densidade:

(a) os pontos iniciais de busca gerados randomicamente; (b) o agrupamento por núcleo de densidade confirma que a distribuição dos pontos é uniforme em todo espaço amostrado; (c) o resultado das otimizações, onde a melhor distribuição corresponde aos valores de 0 a 0.1 é marcada em vermelho; (d) a aplicação do agrupamento por núcleo de densidade para definir uma janela menor, e mais próxima dos valores corretos, no espaço de busca.

\section{Discussões e Conclusões}

As características do problema de otimização, tais como: linearidade, mínimos locais, ambigüidades, e convergência, podem ser reveladas analisando-se a topografia de função objetivo usando os mapas de contorno (Larson, 1999, Kurt, 2007). Se o problema inverso possuir duas incógnitas, a observação dos mapas de contorno (residual function maps) permite definir qual é o grau de complexidade do problema e orientar a escolha do algoritmo de busca. Entretanto para problemas multimodais, como o investigado com 6 incógnitas, não é possível observar o comportamento global da função objetivo usando essa técnica convencional, ou seja, através das fatias (seções transversais) da função, onde apenas dois parâmetros variam e os outros são mantidos fixos.

Mantendo os 4 parâmetros corretos e variando dois parâmetros, nota-se que a ambigüidade revelada pela fatia da função objetivo (Figuras 5-a e 5-b) não é a mesma que a revelada pelo resultado da otimização, representado em forma de mapa de dispersão sobreposto as curvas da função objetivo, para os quais os outros 4 parâmetros resultam da inversão e correspondem a valores diferentes em cada ponto. Observando-se apenas a fatia em forma do mapa de contorno não se enxerga o correto relacionamento dos parâmetros do modelo e se têm a impressão de que não existe forte ambiguidade do contraste de velocidade da onda P. A Figura 5-c ilustra a ambigüidade citada acima no cálculo da curva do coeficiente de reflexão da onda $P$ : os valores foram calculados para dois pontos sobre a faixa de ambigüidade do mapa de dispersão (círculo amarelo e e verde claro na Figura 5-a), e resultam em curvas idênticas de coeficiente de reflexão de onda $\mathrm{P}$. Na Figura 5-d é apenas ilustrado que esse mesmo contraste de velocidade da onda $P$, mantendo-se os outros parâmetros do modelo em seus valores corretos gera uma curva discrepante da correta.

Foram apresentados alguns exemplos da utilidade em se analisar os mapas de dispersão dos resultados de várias otimizações estocásticas. Esse procedimento pode ter as mais variadas aplicações, mas, sobretudo é uma excelente ferramenta para análise dos problemas de inversão não lineares em mais de duas dimensões.

Outra vantagem de usar procedimento Multi-Start é a facilidade de usar computação em paralelo. Cada uma das soluções é otimizada usando um núcleo diferente de cluster em seqüência montando uma matriz com todas as soluções das estimativas dos parâmetros.

\section{Agradecimentos}

Ao CNPq Processo No 483725/2007-2

\section{Referências}

BOKHONOK, O.; DIOGO, A.L.; PRADO, R.L. Estimativa de parâmetros elásticos a partir das amplitudes sísmicas de ondas refletidas P-P P-Sv Sv-P e Sv-Sv: Parte I. Analise do problema de inversão não linear. IV Simpósio Brasileiro de Geofísica, Anais-CDROM.: SBGF, 2010.

BOTEV, Z. I.; GROTOWSKI J. F.; KROESE, D. P. Kernel density estimation via diffusion. Ann. Statist., v. 56, p. 2916-2957, 2010.

ISAAKS, E. H. \& SRIVASTAVA, R. M. An Introduction to Applied Geostatistics, Oxford University Press, 1989.

LARSEN, J.A., AVO Inversion by Simultaneous P-P and P-S Inversion. M.Sc. Thesis, University of Calgary Department of Geology and Geophysics, 1999.

PRICE, W.L., A controlled random search procedure for global optimization. Computer Journal. v. 20, p.367-370, 1979.

PRICE, W.L., Global optimization algorithms by controlled random search. Journal of Optimization Theory and Applications. v. 40, p. 333-348, 1983.

ELIGIUS, M.T., HENDRIX, P.M., GARCIA I., On success rates for controlled random search. J. Global Optim.. v. 21, p. 239-263, 2001. 

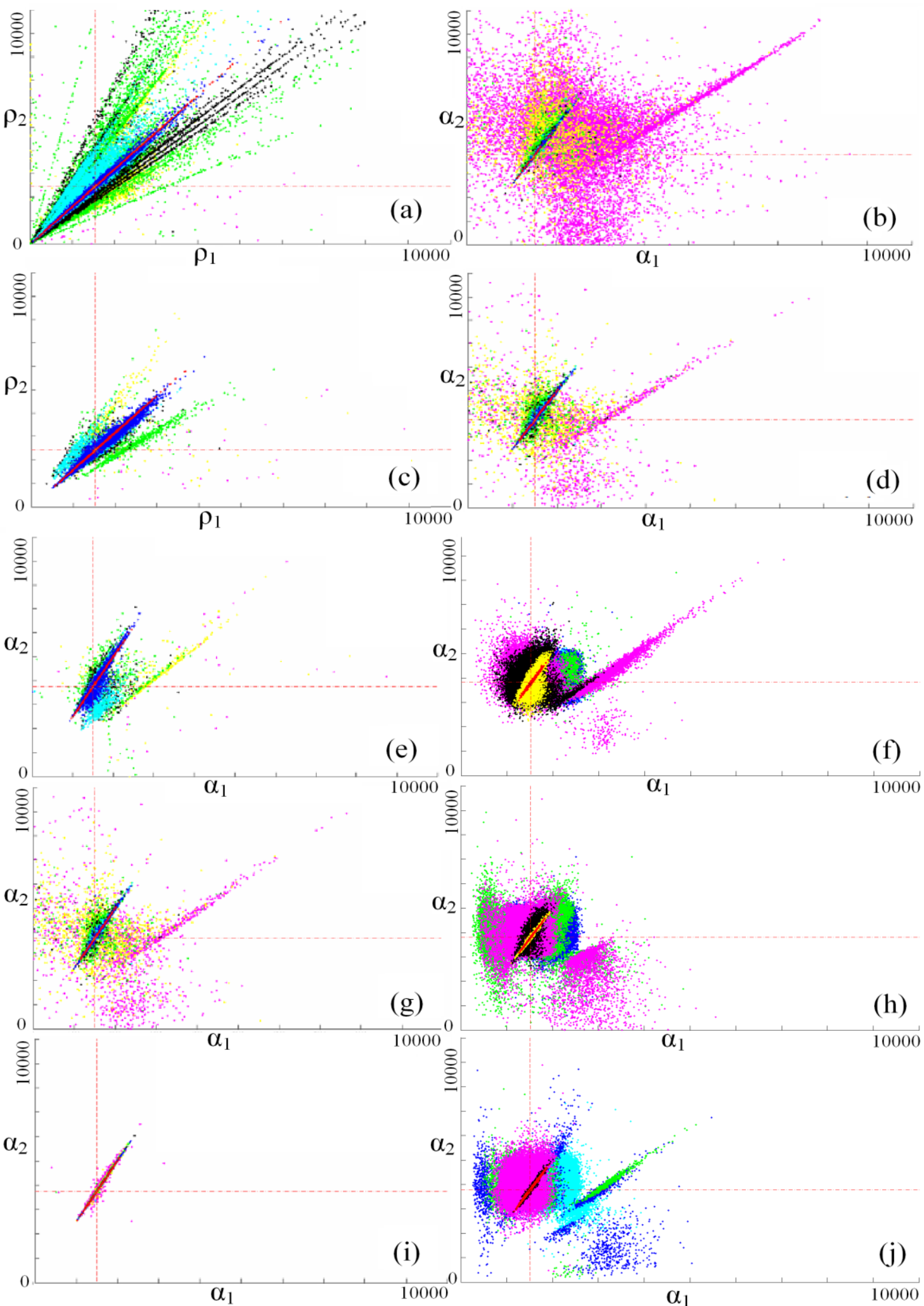

g)

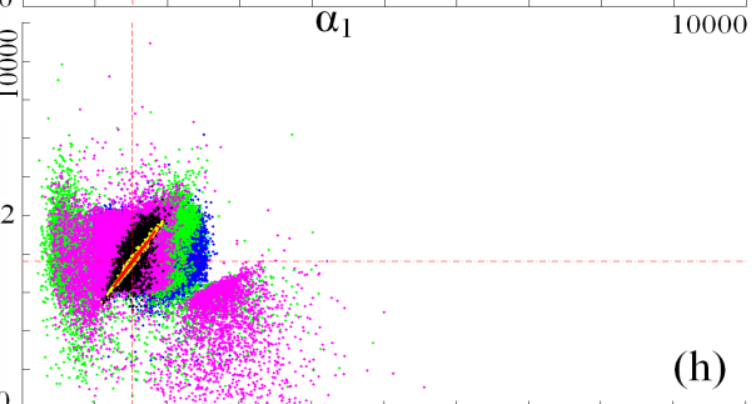

(h)

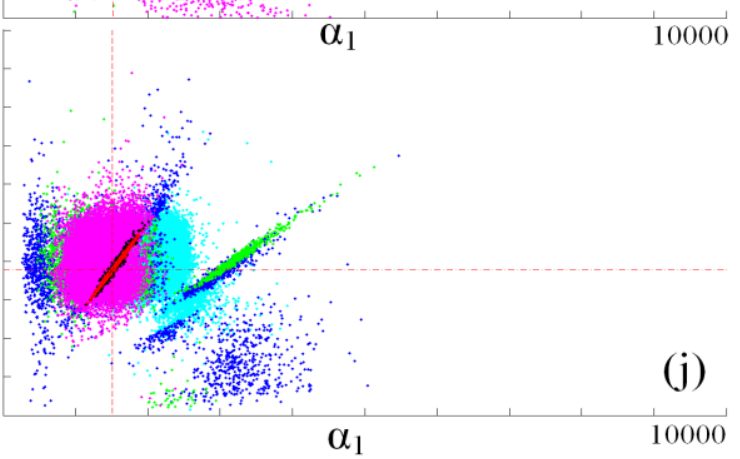

Figura 2 - Exemplos de análise das dispersões para avaliar: (a) e (c) a influência do tamanho do espaço inicial de busca; (b) e (d) a performance de diferentes procedimentos de busca; (e), (g) e (i) a comparação entre diferentes funções objetivo; (f), (h) e (j) o efeito do ruído nos dados. 

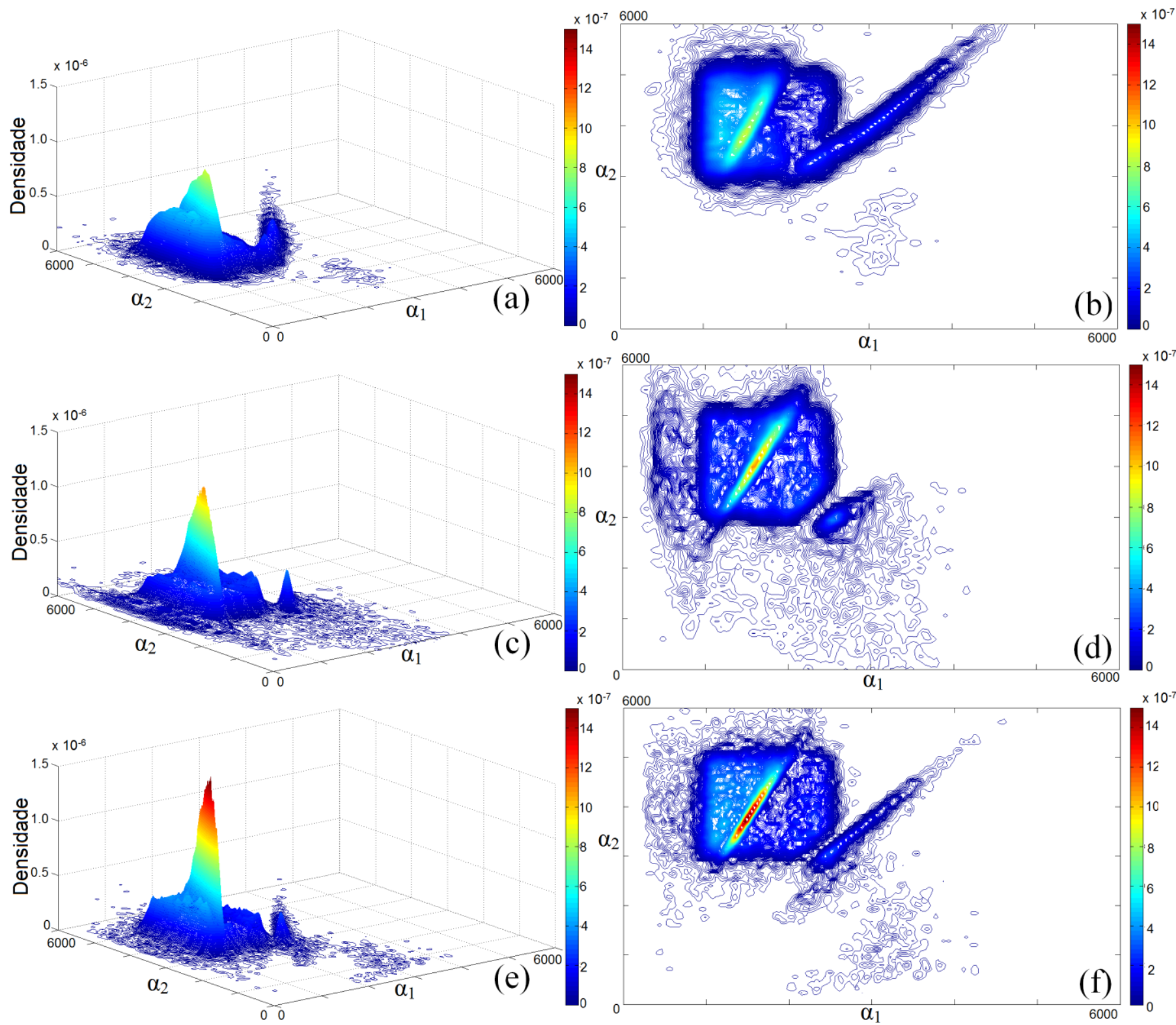

Figura 3 - Gráficos gerados empregando o método de agrupamento por núcleo de densidade aplicado nos mapas de dispersão apresentados nas Figuras 2-f, -h e -j, respectivamente: (a) e (b) PP; (c) e (d) PSV; (e) e (f) PP-PSV (conjunta). 


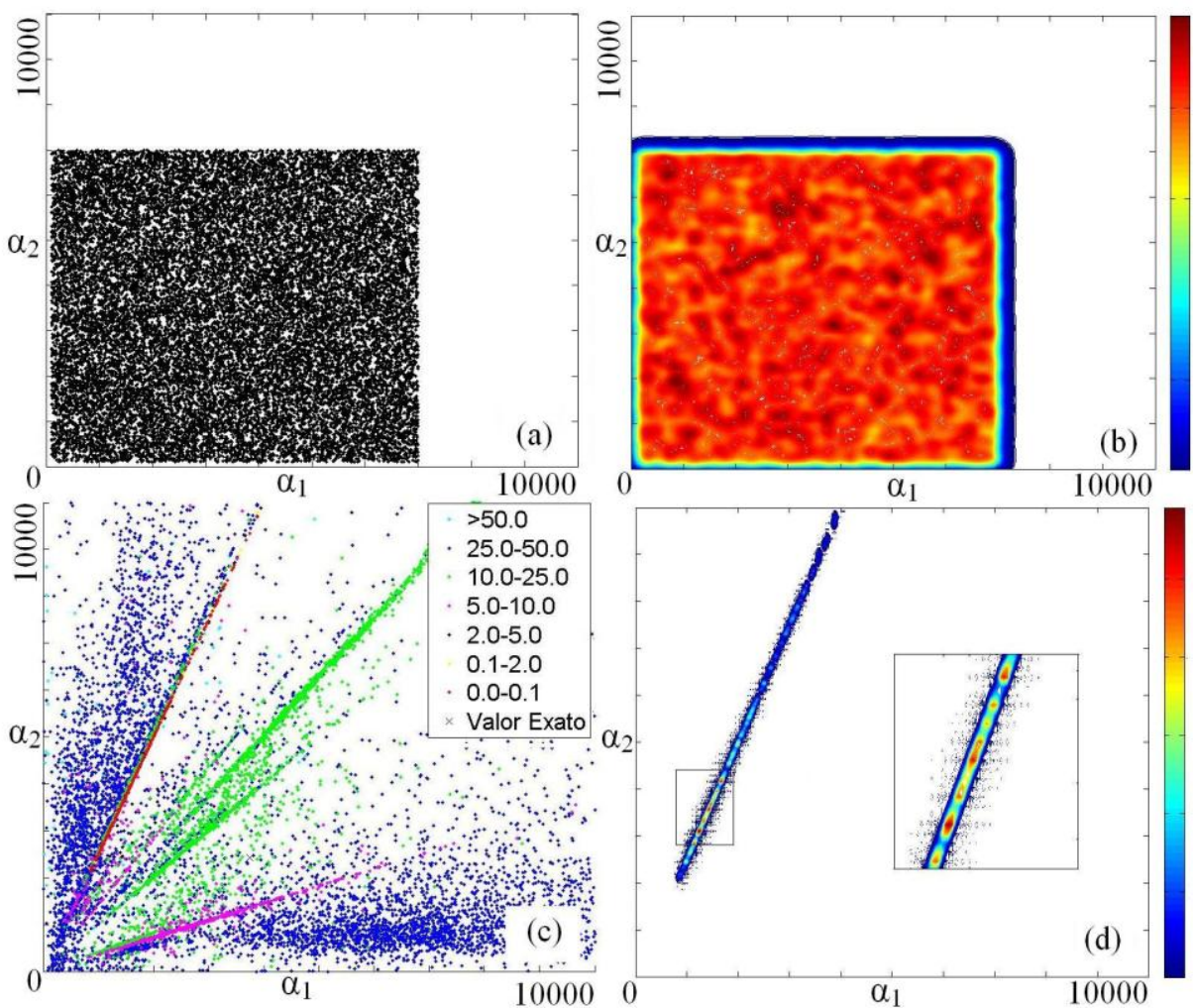

Figura 4 - Ilustração de método de agrupamento por núcleo de densidade para limitar espaço de busca: (a) os pontos iniciais de busca gerados randomicamente num espaço de busca inicial; (b) o agrupamento por núcleo de densidade confirmando a distribuição uniforme dos pontos em espaço de busca inicial; (c) o resultado de otimização, onde a melhor distribuição corresponde aos valores de 0 a 0.1 é marcada em cor vermelha; (d) a redução de espaco de busca.
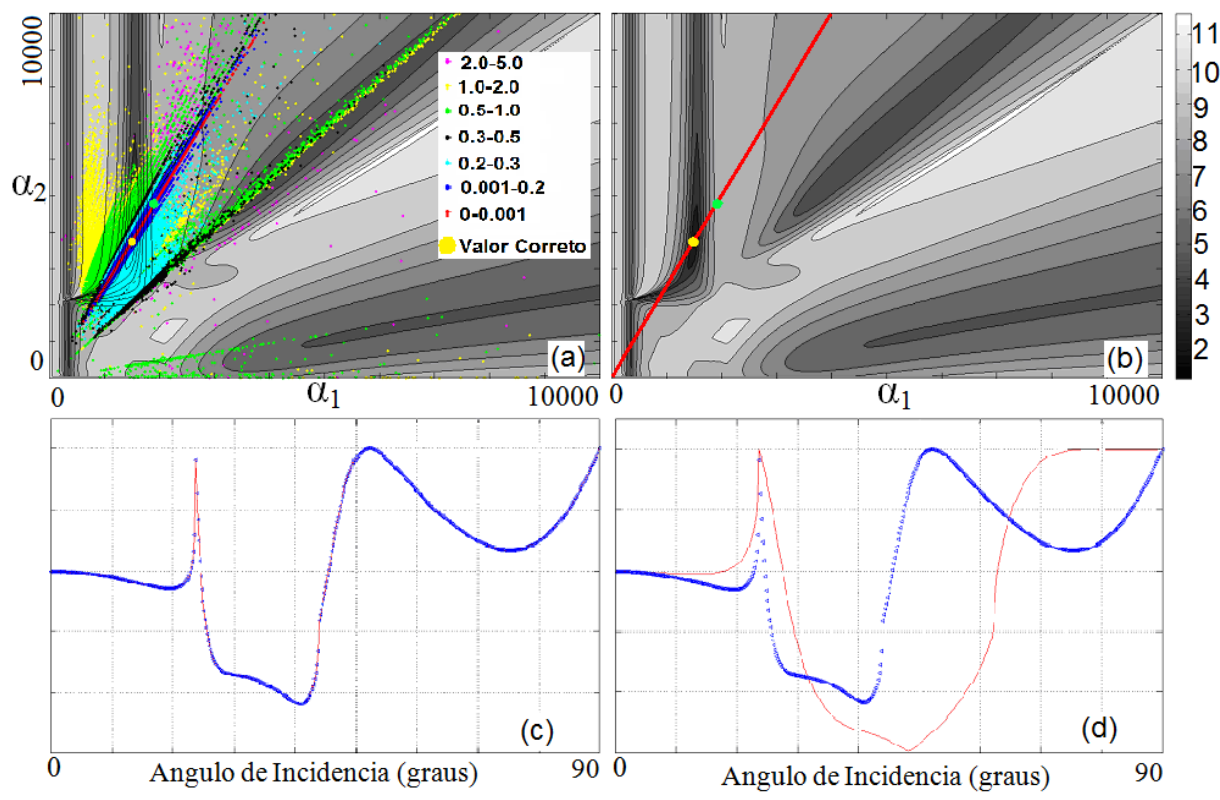

Figura 5 -(a) Mapa de dispersão sobreposto á mapa de contorno de função objetivo; (b) ambigüidade global (linha vermelha) não coincide com ambigüidade que aparece no mapa de contorno; (c) as duas curvas são do coeficiente de reflexão PP, são idênticas e calculadas usando valores que correspondem aos pontos amarelo (modelo) e verde claro (marcados sobre ambigüidade global); (d) curvas do coeficiente de reflexão PP calculadas a partir dos valores que correspondem ao ponto verde claro, tomado: do mapa de dispersão (curva azul) e do mapa de contorno da função objetivo (curva vermelha). 